\title{
La théorie critique de la race en médecine
}

\author{
Citation : CMAJ 2021 May 17;193:E739-41. doi : 10.1503/cmaj.210178-f
}

Voir la version anglaise de l'article ici : www.cmaj.ca/lookup/doi/10.1503/cmaj.210178

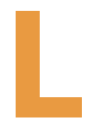
e 4 septembre 2020, le bureau exécutif du président des ÉtatsUnis a publié une note de service à l'intention des agences gouvernementales américaines pour qu'elles cessent de financer toute formation du personnel liée à la théorie critique de la race ou au " privilège blanc », ou à ces deux enjeux, les jugeant « anti-américains » et « sources de division $»^{1}$. Ironiquement, cette publication a fait entrer la théorie critique de la race dans le discours public, et a même fait l'objet d'une discussion lors du premier débat présidentiel américain, où l'éducation basée sur la théorie critique de la race a été décrite comme une "révolution radicale $»^{1}$.

En tant que femmes à la peau noire et brune en médecine, nous avons trouvé dans la théorie critique de la race un langage qui nous permet de mieux comprendre nos expériences. La théorie critique de la race m'a donné (R.Z.) les outils pour fonder le groupe Black Physicians of British Columbia (www.blackphysiciansofbc.ca) et pour contribuer au changement dans mon propre établissement. Elle m'a aidée (M.S.) à « désapprendre » certains des enseignements sur la race que j'ai reçus pendant ma formation médicale, où l'on considérait ce concept comme un fait biologique plutôt que comme une construction sociale qui maintient en place des relations de pouvoir de longue date. La formation médicale ne m'a certainement pas offert les outils conceptuels nécessaires pour aborder le racisme.

La théorie critique de la race est née de longues années de plaidoyers livrés par des avocats noirs et bruns aux ÉtatsUnis, confrontés à des pratiques d'embauche et à des programmes d'études racistes dans les facultés de droit $^{2,3}$. Profondément ancrée dans l'action fondée sur la théorie, la théorie

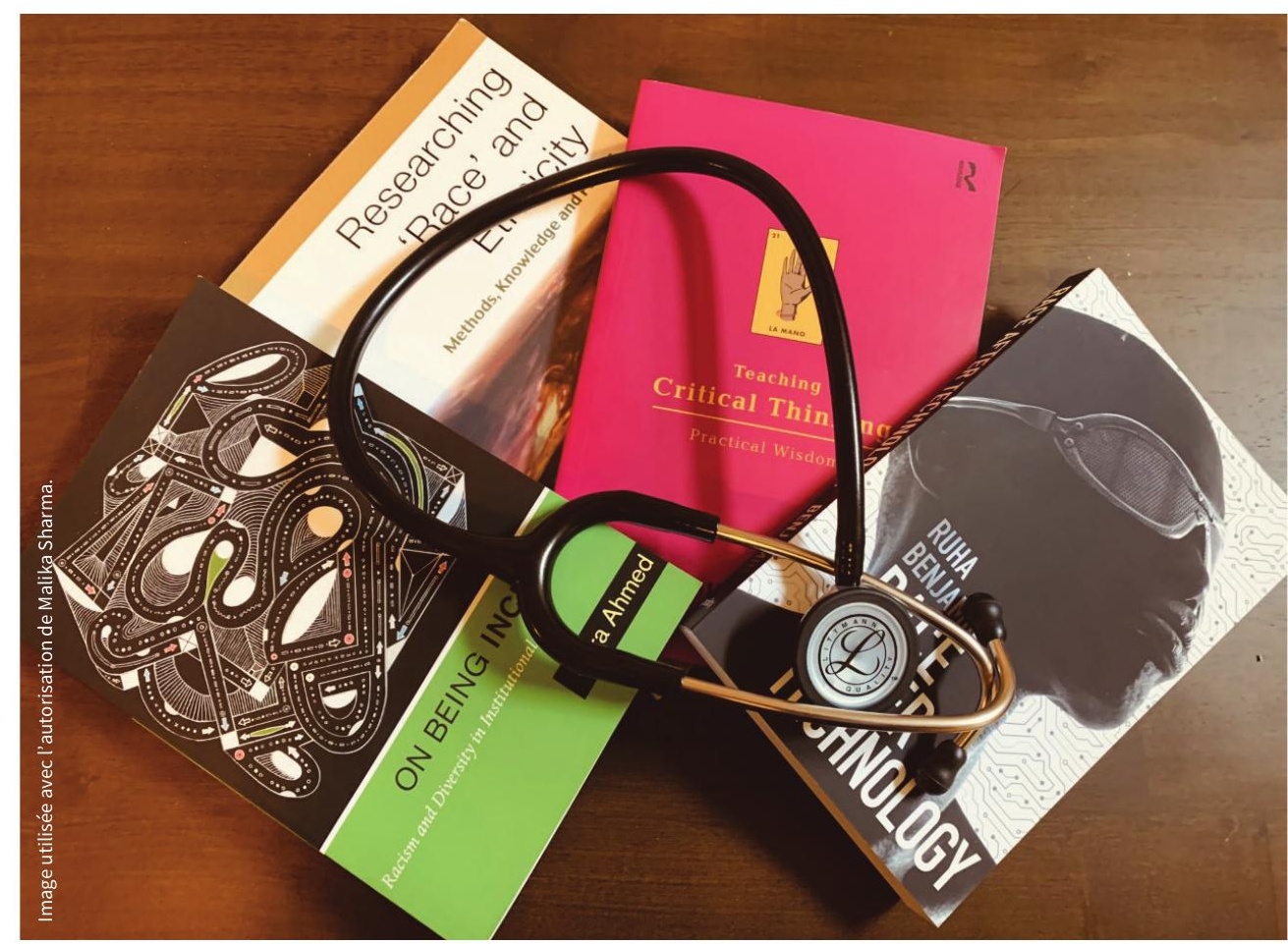

critique de la race peut être utilisée pour comprendre les forces structurelles qui alimentent les inégalités raciales dans la société et pour œuvrer à leur démantèlement $^{2}$. L'étude de la race et du racisme, même sur le plan structurel, ne suffit pas; elle doit être associée à des mesures concrètes à plusieurs niveaux ${ }^{4}$.

La théorie critique de la race s'articule autour de 4 idées principales: la conscience de la race, l'orientation contemporaine, le centrage des marges et la praxie ${ }^{2}$.

La conscience de la race renvoie à une compréhension de la racisation, ou de la création de la « race » en tant que processus social attribuant une signification et une valeur aux différences physiques et culturelles entre les personnes. La conscience de la race nous oblige à examiner ce que nous tenons pour acquis à propos de la race et du racisme, en nous demandant comment ils ont façonné les interactions individuelles et interpersonnelles, ainsi que nos institutions et nos sociétés ${ }^{4}$. En médecine, cela implique une réflexion critique sur ce qui permet de percevoir la race comme un fait biologique, et sur la raison pour laquelle il est si facile d'utiliser la race pour expliquer les différences de santé entre les personnes tout en négligeant les effets des forces politiques et sociales. Le racisme a été intégré à la médecine par le biais de l'objectivité apparente des chiffres (comme les "corrections raciales » pour les mesures physiologiques) et par les détails que nous négligeons chaque jour dans la pratique clinique ${ }^{5}$. La théorie critique de la race nous pousse à réévaluer les pratiques racistes que nous tenons pour acquises et les politiques que nous 
percevons comme « neutres ». Par exemple, le fait d'obtenir les antécédents des patients sans systématiquement faire appel à des services de traduction professionnels en cas de divergence linguistique entre le patient et le fournisseur de soins est une pratique tout à fait inadéquate, mais pourtant largement acceptée. Cette pratique a des répercussions disproportionnées sur les personnes et les familles racisées ${ }^{3}$.

Dans le contexte de la théorie critique de la race, on entend par " centrage des marges » la réorientation de notre attention du groupe dominant vers les groupes qui ont été repoussés dans les marges $^{2}$. Cela suppose de valoriser l'expertise des personnes qui ont été marginalisées et opprimées, en vue de lutter contre les inégalités et de créer de nouvelles solutions.

Un nombre croissant d'études médicales fondées sur la théorie critique de la race, publiées au cours des 10 dernières années, ont mis en lumière les répercussions du racisme sur la manière dont les médecins traitent leurs patients, qu'il s'agisse de soins primaires, de rhumatologie ou d'obstétrique $e^{2,4,6-8}$. Cependant, on a peu discuté des moyens d'intégrer la théorie critique de la race au sein même de la profession médicale.

\section{Éducation médicale}

En présentant la race comme une construction sociale, les enseignants cliniques peuvent favoriser la conscience de la race afin d'éviter de perpétuer des stéréotypes nuisibles, comme la croyance que l'organisme des Noirs est plus " tolérant à la douleur ", une idée fausse qui a entraîné des recommandations d'analgésiques inappropriées pour de nombreux patients ${ }^{9}$. Les programmes des facultés de médecine peuvent analyser de manière critique la façon dont la " race » est utilisée comme un marqueur indirect des racines structurelles des problèmes de santé, telles que la pauvreté et l'accès différentiel aux soins de santé et à l'éducation. Cependant, l'utilisation de la race comme marqueur indirect sans tenir compte des problèmes structurels ne peut que renforcer les hypothèses préjudiciables et erronées.
Les cliniciens peuvent « ramener les marges au centre » en recherchant activement et en valorisant les connaissances et les points de vue des patients, des apprenants et des éducateurs racisés. Récemment, des étudiants en médecine ont démontré leur capacité à apporter des changements dans le cadre d'un mouvement d'envergure mondiale visant à mieux représenter les personnes racisées dans le matériel d'enseignement de la dermatologie $\mathrm{e}^{10}$.

\section{Soins cliniques}

Il est essentiel de procéder à un examen critique du manque de données probantes (et de la véracité de celles qui existent) à l'appui des décisions cliniques fondées sur la race. On peut par exemple s'interroger sur la logique qui sous-tend la recommandation de diurétiques aux patients canadiens provenant d'Afrique de l'Est, sur la base d'essais cliniques impliquant des participants afroaméricains principalement originaires d'Afrique de l'Ouest ${ }^{11}$.

Les fortes disparités raciales observées dans la morbidité et la mortalité liées à la COVID-19 et dans l'accès aux vaccins contre le SRAS-CoV-2 ont mis en évidence les obstacles systémiques auxquels certains groupes font face pour accéder au système médical. La praxie, ou l'action fondée sur la théorie, incite les cliniciens à aller au-delà de la simple reconnaissance du racisme et à intervenir pour éliminer les disparités en santé fondées sur la race. Des campagnes de santé publique ciblées, par exemple la récente initiative du Black Doctors COVID Consortium, qui vise à vacciner les membres de la communauté noire qui travaillent de longs quarts ou qui ont de la difficulté à s'inscrire en ligne, peuvent contribuer à réduire les obstacles aux soins médicaux et à rétablir progressivement la confiance dans le système de santé ${ }^{12}$.

\section{Racisme en milieu de travail}

Les travailleurs de la santé racisés sont victimes de microagressions et d'autres formes de racisme ordinaire au travail. En Ontario, $70 \%$ des médecins noirs sont fréquemment victimes de discrimination raciale; par exemple, on les confond avec le personnel d'entretien, ils reçoivent des commentaires non sollicités sur leurs cheveux, ils sont écartés pour des promotions et leurs compétences sont remises en question ${ }^{11}$. Cette "banalité » du racisme ordinaire fait peser un lourd fardeau sur les médecins racisés, qui doivent prouver à leurs collègues, à leurs superviseurs et aux instances disciplinaires comment le racisme les affecte personnellement au travail. La théorie critique de la race incite à réfléchir aux fondements structurels qui sous-tendent ces expériences quotidiennes et à agir pour y remédier. Plutôt que de considérer le racisme ordinaire comme des incidents mineurs isolés, la théorie critique de la race invite à mettre l'accent sur les expériences des étudiants et des médecins racisés et à passer de l'intention à l'action. Elle peut conduire à des mesures telles que l'examen et la modification des pratiques d'embauche et des programmes de formation, ou encore la création de communautés de soutien pour favoriser des milieux de travail sûrs et inclusifs.

\section{Recherche}

Des exemples historiques tels que l'étude de Tuskegee sur la syphilis aux États-Unis, les expériences sur la malnutrition menées sur des enfants autochtones dans des pensionnats au Canada et les recherches fondées sur l'hypothèse que les Autochtones étaient physiologiquement "prédisposés » à la tuberculose n'ont guère contribué à inspirer la confiance des personnes racisées dans la recherche médicale. La logique médicale continue d'associer la "vulnérabilité » à la maladie des personnes racisées à leur organisme, comme en témoignent les récentes tentatives d'associer des polymorphismes génétiques à des taux plus élevés de COVID-19 chez les Noirs américains ${ }^{13}$. La théorie critique de la race encourage une responsabilité partagée qui consiste à aller au-delà de ces modèles réductionnistes de la santé et de la maladie et à examiner les forces sociales qui alimentent les iniquités raciales. 


\section{Conclusion}

La profession médicale est de plus en plus appelée à lutter contre le racisme et à promouvoir l'équité, la diversité et l'inclusion. La théorie critique de la race, qui a récemment été mise à l'avant-plan du débat public, représente un puissant outil de changement. En effet, le moment est venu de mener cette "révolution radicale».

\section{Rahel Zewude MD}

Département de médecine, Université de la Colombie-Britannique, Vancouver, C.-B. Malika Sharma MD MEd

Division des maladies infectieuses, Département de médecine, Hôpital St. Michael; professeure adjointe, Université de Toronto, Toronto, Ont.

\section{Références}

1. Vought R. M-20-34: Memorandum for the heads of executive departments and agencies. Washington (DC): Executive Office of the President, Office of Management and Budget; 2020 Sept. 4. Accessible ici : www.whitehouse.gov/wp-content /uploads/2020/09/M-20-34.pdf (consulté le 9 oct. 2020).
2. Ford CL, Airhihenbuwa CO. Critical Race Theory, race equity, and public health: toward antiracism praxis. Am J Public Health 2010;100(Suppl 1): S30-5.

3. Bell DA. David C. Baum Memorial Lecture: Who's afraid of Critical Race Theory? 1995 U. Ill. Law Rev 99994. Champaign (IL): Illinois College of Law; 1995:893.

4. Ford CL, Airhihenbuwa CO. The public health critical race methodology: praxis for antiracism research. Soc Sci Med 2010;71:1390-8.

5. Vyas DA, Eisenstein LG, Jones DS. Hidden in plain sight: reconsidering the use of race correction in clinical algorithms. N Engl J Med 2020;383: 874-82.

6. Williams JN, Ford CL, Morse M, et al. Racial disparities in rheumatology through the lens of Critical Race Theory. Rheum Dis Clin North Am 2020;46:605-12.

7. Adebayo CT, Parcell ES, Mkandawire-Valhmu L, et al. African American Women's maternal healthcare experiences: a Critical Race Theory perspective. Health Commun 2021 Feb. 18 [cyberpublication avant impression]. doi: 10.1080/10410236.2021.1888453.

8. Cunningham A, Crittendon D, Konys C, et al. Critical Race Theory as a lens for examining primary care provider responses to persistentlyelevated HbA1c. J Natl Med Assoc 2020 Dec. 17 [cyberpublication avant impression]. doi: 10.1016/j.jnma.2020.11.012.

9. Hoffman KM, Trawalter S, Axt JR, et al. Racial bias in pain assessment and treatment recommendations, and false beliefs about biological differences between blacks and whites. Proc Natl Acad Sci U S A 2016;113:4296-301.
10. Medical schools must include BAME representation in clinical teaching. Change.org. Accessible ici : www.change.org/p/gmc-medical-schools-must -include-bame-representation-in-clinical-teaching ?signed=true (consulté le 25 mars 2021).

11. Mpalirwa J, Lofters A, Nnorom O, et al. Patients, pride, and prejudice: exploring Black Ontarian physicians' experiences of racism and discrimination. Acad Med 2020;95:S51-7.

12. Ralph P. Thousands of Philly residents inoculated at 24-hour COVID-19 vaccine clinic. PhillyVoice 2021 Feb. 21. Accessible ici : www.phillyvoice.com/ black-doctors-covid-19-consortium-vaccination -site-philadelphia/ (consulté le 16 mars 2021).

13. Phillips N, Park I-W, Robinson JR, et al. The perfect storm: COVID-19 health disparities in US Blacks. J Racial Ethn Health Disparities 2020 Sept. 23 [cyberpublication avant impression]. doi: 10.1007/s40615-020-00871-y.

Cet article a été révisé par des pairs.

Intérêts concurrents : Aucun déclaré.

Propriété intellectuelle du contenu : Il s'agit d'un article en libre accès distribué conformément aux modalités de la licence Creative Commons Attribution (CC BY-NC-ND 4.0), qui permet l'utilisation, la diffusion et la reproduction dans tout médium à la condition que la publication originale soit adéquatement citée, que l'utilisation se fasse à des fins non commerciales (c.-à-d., recherche ou éducation) et qu'aucune modification ni adaptation n'y soit apportée. Voir : https://creativecommons.org/ licenses/by-nc-nd/4.0/deed.fr. 